\title{
Substrate influence on the properties of doped thin silicon layers grown by Cat-CVD
}

\author{
D. Soler ${ }^{1}$, M. Fonrodona ${ }^{1}$, C. Voz ${ }^{2}$, J.M. Asensi ${ }^{1}$, J. Bertomeu ${ }^{1}$ and J. Andreu ${ }^{1}$
}

${ }^{1}$ Dept. Física Aplicada i Òptica, Universitat de Barcelona, Av. Diagonal 647, 08028

Barcelona, Spain.

${ }^{2}$ Dept. Enginyeria Electrònica, Universitat Politècnica de Catalunya, Gran Capità s/n, Mòdul C4, 08034 Barcelona, Spain

\begin{abstract}
We present structural and electrical properties for $\mathrm{p}$-type and n-type layers grown close to the transition between a-Si:H and nc-Si:H onto different substrates: Corning 1737 glass, $\mathrm{ZnO}: \mathrm{Al}$ coated glass and stainless steel. Both crystalline fraction and dark conductivity at room temperature exhibited a transition from a-Si:H to nc-Si:H, which was clearly dependent on the substrate used. Stainless steel seemed to promote crystalline growth for n-type layers whereas glass seemed to enhance crystallinity for p-type material. Electrical conduction in the direction perpendicular to the substrate seemed to be mainly determined by the contacts. In this direction, n-type layers showed a lower resistance value than p-type ones, showing a better contact between the layer and the substrate.
\end{abstract}

\section{Keywords}

Catalytic chemical vapour deposition, Nanocrystalline silicon, Amorphous silicon, doping 


\section{Introduction}

Silicon thin films obtained close to the transition between nanocrystalline silicon (nc-Si:H) and amorphous silicon (a-Si:H) have recently been proved as specially suitable when applied to solar cells obtained by Catalytic Chemical Vapour Deposition (Cat-CVD), also called Hot-Wire CVD (HWCVD) [1]. The microstructure of silicon thin films prepared by Cat-CVD can be considerably influenced by slight changes in the deposition parameters. In particular, the transition from a-Si:H to nc-Si:H can be observed in a narrow range of hydrogen dilution $\left(\mathrm{D}_{\mathrm{H}}\right)[2,3]$. Furthermore, the above mentioned transition has also been proved to depend on the properties of the substrate on which the sample is grown for both plasma enhanced CVD (PE-CVD) and Cat-CVD [4, 5].

Different solar cell configurations (p-i-n/n-i-p) require controlled growth of both p-type and n-type layers on different substrates. p-i-n cells are usually deposited onto transparent conductive oxide (TCO) whereas n-i-p cells are commonly grown on metallic substrates. Thus, the substrate influence on the microstructure and electrical properties of the doped layers has to be carefully considered, especially when dealing with deposition conditions close to the transition zone. Moreover, p-type and n-type layers used in solar cells are typically very thin so the initial stages of growth may have a very important influence on the final properties of the layer.

In this study we focus on the low-temperature deposition of doped layers in the range of $100 \mathrm{~nm}$ close to the transition zone by Cat-CVD on different substrates. We will show that 
p-type and n-type films exhibit different behaviour regarding the microstructural and electrical properties.

\section{Experimental}

Doped thin silicon layers with thicknesses in the range of $100 \mathrm{~nm}$ were simultaneously deposited onto Corning 1737 glass, stainless steel (SS) and $\mathrm{ZnO}: \mathrm{Al}$ coated glass in a CatCVD set-up described elsewhere [6]. The dissociation of the gases was achieved by means of a tantalum filament resistively heated up to temperatures around $1800^{\circ} \mathrm{C}$. A process pressure of $3 \times 10^{-2}$ mbar was used while a substrate temperature of $200^{\circ} \mathrm{C}$ for $\mathrm{n}$-type layers and $125^{\circ} \mathrm{C}$ for $\mathrm{p}$-type samples were selected. The transition from a-Si:H to nc-Si:H was achieved by means of varying the hydrogen dilution $\left(\mathrm{D}_{\mathrm{H}}=\phi_{\mathrm{H} 2} / \phi_{\mathrm{H} 2}+\phi_{\mathrm{SiH} 4}\right)$ between $85 \%$ and $96 \%$. $\phi_{\mathrm{SiH} 4}$ was kept constant at $4 \mathrm{sccm}$, while $\phi_{\mathrm{H} 2}$ was varied between $16 \mathrm{sccm}$ and $102 \mathrm{sccm}$. Two series of p-type and n-type layers were deposited by adding certain amounts of diborane and phosphine to the silane-hydrogen mixture. A gas phase atomic ratio of $1 \%$ was chosen for the n-type samples whereas the atomic ratio for p-type layers was $5 \%$.

The thickness of the samples was measured with a Dektak profilometer. The microstructure of the layers was analysed by Raman spectroscopy by using a Jobin-Yvon T64000 spectrometer, and the crystalline fraction $\left(\mathrm{X}_{\mathrm{c}}\right)$ was deduced by deconvoluting the amorphous and crystalline contributions to the TO-phonon band [7]. Coplanar electrical measurements were performed after evaporating $\mathrm{Cr} / \mathrm{Al}$ stripes $1 \mathrm{~mm}$ apart on the samples deposited onto glass substrates. Dark conductivity vs. temperature was measured in order 
to determine the activation energy $\left(\mathrm{E}_{\mathrm{A}}\right)$. On the other hand, $\mathrm{Cr} / \mathrm{Al} / \mathrm{Au}$ circular electrodes (290 $\mu \mathrm{m}$ diameter) were evaporated on the samples grown onto SS and $\mathrm{ZnO}: \mathrm{Al}$ coated glass. These electrodes allowed us to analyse the electrical behaviour of the doped samples in the direction perpendicular to the substrate by measuring the voltage vs. current curve. Samples were dry-etched with a plasma of $\mathrm{CF}_{4}$ diluted into oxygen $(20 \%)$ at a process pressure of $0.4 \mathrm{mbar}$ and RF power of $15 \mathrm{~mW} / \mathrm{cm}^{2}$. Four probes were used to minimize effects arising from the electrode-probe contact.

\section{Results and discussion}

Structural and electrical properties of doped layers exhibited a clear dependence on $\mathrm{D}_{\mathrm{H}}$ and substrate properties. Moreover, different behaviour was observed for p-type and n-type layers.

(a) Structural properties

$\mathrm{X}_{\mathrm{c}}$ of both p-type and n-type layers can be seen in Fig. 1 as a function of $\mathrm{D}_{\mathrm{H}}$. On one hand, SS seemed to promote the crystalline growth when dealing with n-type samples whereas glass and $\mathrm{ZnO}: \mathrm{Al}$ coated glass exhibited a very similar trend. Thus, a $\mathrm{D}_{\mathrm{H}}$ value around $85 \%$ was necessary for all substrates in order to reach a measurable crystalline fraction. However, $X_{c}$ values obtained for samples deposited onto SS remained higher than those observed both on glass and $\mathrm{ZnO}: \mathrm{Al}$ coated glass for all studied dilutions. On the other hand, $\mathrm{X}_{\mathrm{c}}$ values for $\mathrm{p}$-type samples exhibited a different behaviour. Under present deposition conditions, higher $\mathrm{D}_{\mathrm{H}}$ values were necessary for p-type layers. Only $\mathrm{D}_{\mathrm{H}}$ above 
95\% led to significant crystalline fractions. Contrary to the results obtained when dealing with n-type layers, an increased $X_{c}$ was observed on those samples deposited onto glass. In fact, $X_{c}$ values were only detected on glass for those samples obtained with $D_{H}=95 \%$, whereas $\mathrm{D}_{\mathrm{H}}$ around $96.2 \%$ were necessary to observe any crystalline fraction for samples deposited on either SS or $\mathrm{ZnO}: \mathrm{Al}$ coated glass.

(b) Electrical properties

Electrical conductivities corresponding to both p-type and n-type layers are shown in Fig.2. Coplanar measurements performed on those samples deposited onto glass exhibited a clear correlation with structural results obtained by Raman spectroscopy. Coplanar conductivity at room temperature $\left(\sigma_{/ /}\right)$exhibited a clear transition for n-type samples around $\mathrm{D}_{\mathrm{H}}=85 \%$. $\sigma_{/ /}$values between 2.3 and $4 \mathrm{~S} / \mathrm{cm}$ were observed for n-type layers deposited with $\mathrm{D}_{\mathrm{H}}$ between $90 \%$ and $95 \%$. These samples exhibited $\mathrm{E}_{\mathrm{A}}$ values between 0.10 and $0.06 \mathrm{eV}$. On the other hand, $\mathrm{D}_{\mathrm{H}}$ above $95 \%$ was necessary when dealing with p-type samples to achieve $\sigma_{/ /}$values corresponding to those typically observed in nc-Si:H. Thus, $\sigma_{/ /}$values between 2.2 and $4.6 \mathrm{~S} / \mathrm{cm}$ were obtained for samples deposited using $\mathrm{D}_{\mathrm{H}}$ of $95 \%$ and $96.2 \%$. These samples exhibited $\mathrm{E}_{\mathrm{A}}$ values of 0.05 and $0.03 \mathrm{eV}$.

The evolution of $X_{c}$ shown in Fig.1 and $\sigma_{/ /}$observed in Fig.2 clearly indicated that higher $D_{H}$ was necessary for $\mathrm{p}$-type layers than for n-type ones to reach nc-Si:H properties. The high diborane concentration seemed to be responsible for this behaviour. It is known that diborane tends to increase the amorphous character of samples [6], so a decrease in $\mathrm{D}_{\mathrm{H}}$ to reach nc-Si:H properties could be expected after lowering the diborane concentration. The 
evolution of $\sigma_{/ /}$presented in Fig.2 for amorphous samples exhibits the same trend for both p-type and n-type layers. Thus, samples deposited just before the transition to nc-Si:H $\left(D_{H}=85 \%\right.$ for $n$-type material and $D_{H}=92.5 \%$ for $p$-type layers) exhibited the lowest $\sigma_{/ /}$ values while it increased as $\mathrm{D}_{\mathrm{H}}$ was lowered. These results could be attributed to a worsening of the a-Si:H properties as the deposition conditions approached those that lead to nc-Si:H. This result could be provoked by an increase in the structural disorder and the porosity of a-Si:H samples as $\mathrm{D}_{\mathrm{H}}$ was increased.

Measurements performed in the direction perpendicular to the substrate on those samples deposited onto SS and $\mathrm{ZnO}$ :Al coated glass allowed us to obtain some information of the properties of the doped layers and the corresponding interfaces. Voltage vs. current measurements were performed between $-25 \mathrm{~mA} / \mathrm{cm}^{2}$ and $25 \mathrm{~mA} / \mathrm{cm}^{2}$ so that standard conditions under which solar cells work could be evaluated. The obtained voltage vs. current curves exhibited a linear behaviour for both p-type and n-type samples on both SS and ZnO:Al coated glass. This result indicated an ohmic behaviour of the corresponding junctions. This fact allowed us to determine the resistance from which the conductivity $\left(\sigma_{\perp}\right)$ could be extracted. Some of the results can also be observed in Fig.2. It is important to note that the conductivity perpendicular to the substrate presented in figure 2 has been evaluated attributing a homogeneous conductivity to the layer, without any effect of the interfaces. In fact, the big disagreement between these values and the ones determined from the conductivity parallel to the substrate indicated that these conductivity values did not correspond to the layer itself. The conduction perpendicular to the substrate seemed to be mainly controlled by the electrical transport in the barriers created in the junction between the semiconductor and the substrates. In fact, the resistance of the 
semiconductor in the parallel direction was approximately evaluated by considering the same conductivity in both directions. The extracted value could be neglected when compared with the measured resistance. It seemed clear that n-type material exhibited better contact properties than p-type layers, regardless the crystalline fraction. The contact resistance of n-type layers remained almost constant for both amorphous and nanocrystalline material and for both SS and ZnO:Al coated glass. In Fig.3, Raman spectra for n-type layers grown with $\mathrm{D}_{\mathrm{H}}=80 \%$ and $\mathrm{D}_{\mathrm{H}}=90 \%$ are presented on SS and ZnO:Al coated glass. Samples grown with $\mathrm{D}_{\mathrm{H}}=80 \%$ were amorphous for all substrates whereas layers obtained with $\mathrm{D}_{\mathrm{H}}=90 \%$ exhibited $\mathrm{X}_{\mathrm{c}}$ values around $75 \%$.

Conductivity measurements shown in Fig. 2 for the direction perpendicular to the substrate $\left(\sigma_{\perp}\right)$ allowed us to obtain a value for the contact resistance. These values were clearly dependent on the type of dopant. Thus, contact resistances between 1 and $3 \Omega \cdot \mathrm{cm}^{2}$ were obtained for $\mathrm{p}$-type samples whereas n-type layers exhibited values close to $0.05 \Omega \cdot \mathrm{cm}^{2}$. pi-n solar cells usually present a TCO/p junction whereas n-i-p cells can be grown on SS [8] where a SS/n junction is observed. Assuming a typical current density of $20 \mathrm{~mA} / \mathrm{cm}^{2}$, a potential drop between 20 and $60 \mathrm{mV}$ for the $\mathrm{TCO} / \mathrm{p}$ junction and around $1 \mathrm{mV}$ for the SS/n one was obtained.

\section{Conclusions}

Thin silicon material obtained close to the transition between a-Si:H and $\mathrm{nc}-\mathrm{Si}: \mathrm{H}$ is a very promising material for thin-film solar cells. 
We have shown that both structural and electrical properties of p-type and n-type layers obtained by Cat-CVD depend on the substrate used. SS substrates seemed to promote crystalline growth for n-type material whereas glass enhanced crystallinity of p-type layers.

Electrical behaviour perpendicular to the substrate seemed to be mainly dependent on the interface properties. All samples exhibited a linear trend showing the ohmic character of the junction. The resistance depended mainly on the used dopant, while structural and electrical properties of the obtained layers did not play a critical role.

\section{Acknowledgments}

This work has been financed by the Spanish Government (MAT2001-3451-C03-01). The authors want to thank the Scientific-Technical Services of the UB for the Raman Spectroscopy measurements.

\section{References}

[1] S. Klein, F. Finger, R. Carius, B. Rech, L. Houbern, M. Luysberg, M. Stutzman, Mat. Res. Soc. Symp. Proc. 715 (2002), in press.

[2] P. Alpuim, V. Chu, J.P. Conde, J. Appl. Phys. 86 (1999) 3812.

[3] D. Han, G. Yue, D. Lorentzen, J. Lin, H. Habuchi, Q. Wang, J. Appl. Phys. 87 (2000) 1882.

[4] M. Kondo, Y. Toyoshima, A. Matsuda, K. Ikuta, J. Appl. Phys. 80 (10) (1996) 6061. 
[5] S. Klein, J. Wolff, F. Finger, R. Carius, H. Wagner, M. Stutzman, Jpn. J. Appl. Phys. 41 (2002) L10.

[6] C. Voz, D. Peiró, J. Bertomeu, D. Soler, M. Fonrodona, J.Andreu, Mater. Sci. Eng. B $69 / 70(2000) 278$.

[7] Z. Iqbal, S. Veprek, A.P. Webb, P. Capezzuto, Solid State Commun. 37 (1981) 993.

[8] R.E.I. Schropp, J.K. Rath, IEEE Transactions on Electronic Devices 46 (1999) 2069. 


\section{Figure captions}

Fig.1. Crystalline fractions $\left(\mathrm{X}_{\mathrm{c}}\right)$ for $\mathrm{p}$-type and n-type layers deposited simultaneously onto different substrates as a function of hydrogen dilution $\left(\mathrm{D}_{\mathrm{H}}\right)$.

Fig. 2. Electrical properties at room temperature for n-type and p-type layers. Glass measurements refer to coplanar conductivity whereas SS and $\mathrm{ZnO}$ :Al measurements refer to conductivity in the perpendicular direction.

Fig. 3. Raman spectra for n-type layers obtained with $\mathrm{D}_{\mathrm{H}}=80 \%$ and $\mathrm{D}_{\mathrm{H}}=90 \%$ on SS and $\mathrm{ZnO}: \mathrm{Al}$ coated glass. 


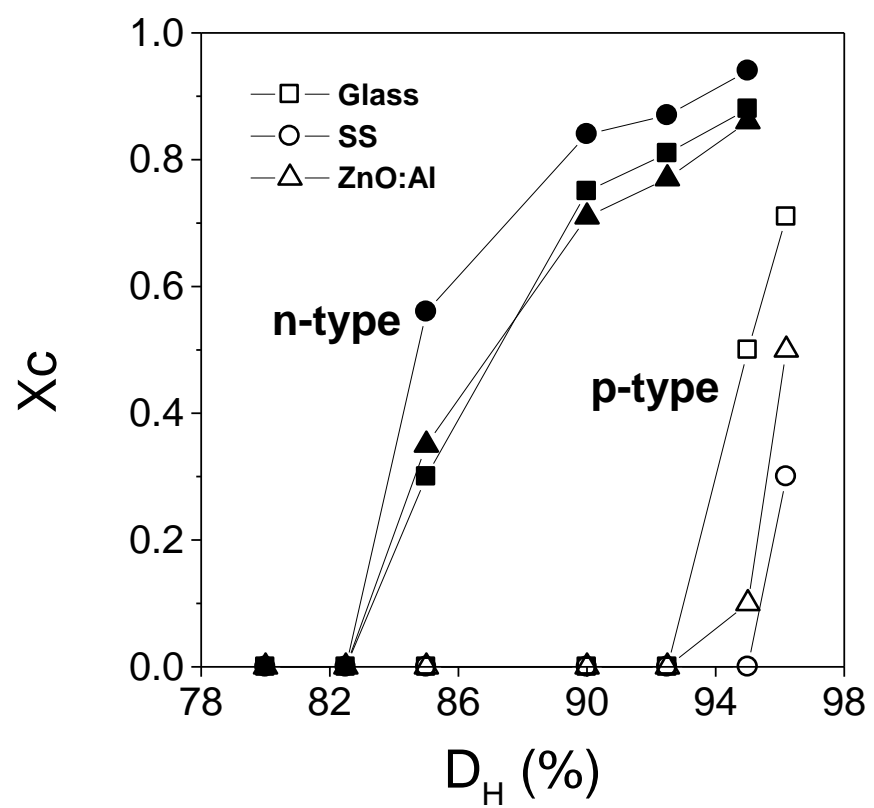

Figure 1 


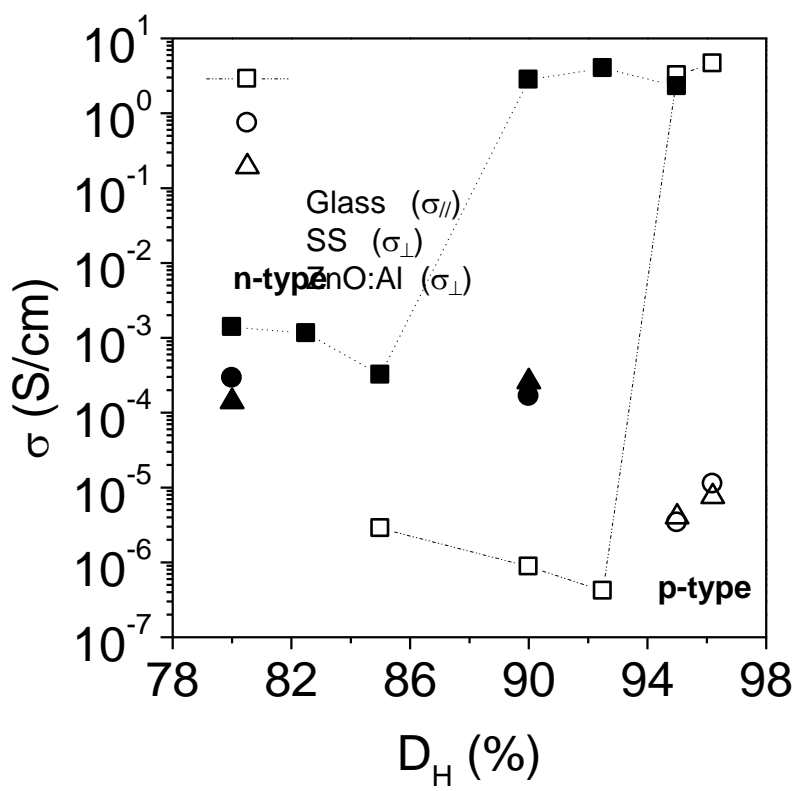

Figure 2 


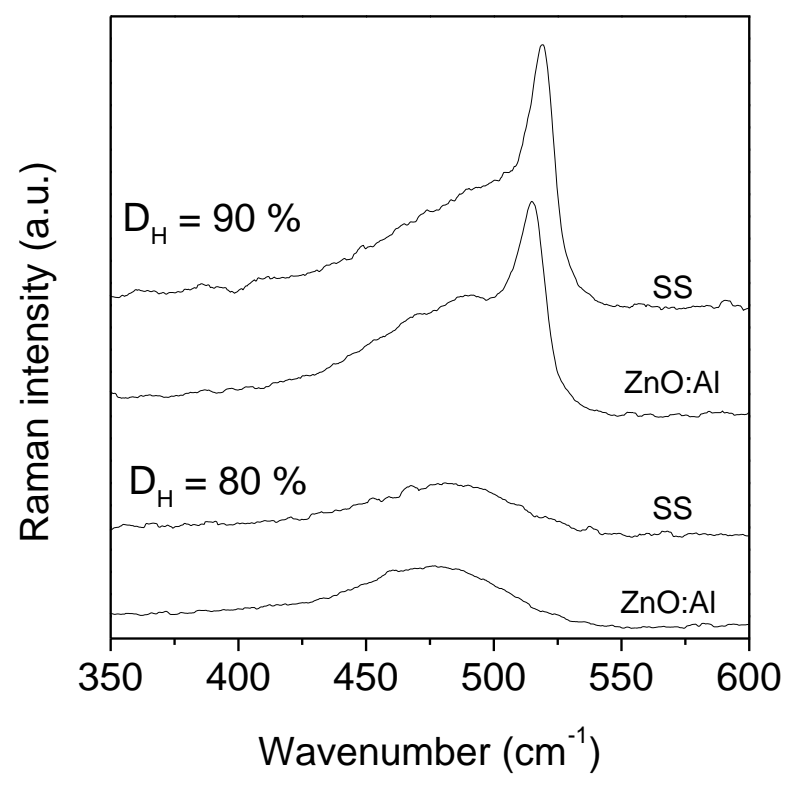

Figure 3 